\title{
Competition between fluctuations and disorder in frustrated magnets
}

\author{
Julien Serreat* and Matthieu Tissiel甘 \\ Institut für Theoretische Physik, Universität Heidelberg, Philosophenweg 16, 69120 Heidelberg, Germany
}

(Dated: November 23, 2018)

\begin{abstract}
We investigate the effects of impurities on the nature of the phase transition in frustrated magnets, in $d=4-\epsilon$ dimensions. For sufficiently small values of the number of spin components, we find no physically relevant stable fixed point in the deep perturbative region $(\epsilon \ll 1)$, contrarily to what is to be expected on very general grounds. This signals the onset of important physical effects.
\end{abstract}

PACS numbers: 75.40.Cx, 11.10.Hi

The influence of disorder in solid state physics is a very important problem. In many cases, it is expected that inhomogeneities, such as impurities, defects in the lattice structure, etc. may induce a completely different behavior in the system. Let us discuss two such situations in the context of phase transitions, which we shall be concerned with in this report. In systems undergoing second order phase transitions, disorder most often changes a divergence in the specific heat at the critical temperature into a cusp. The archetype of such a situation is the $d=3$ Ising model, for which both experimental and theoretical works show that the critical exponent $\alpha$ changes from positive to negative when disorder is added (for a review, see Ref. 1). This is well understood, thanks to the Harris criterion, $\frac{2}{2}$ which states that for a large class of disordered systems, $\frac{3}{,}$ the critical exponent $\nu_{\mathrm{d}}-$ which governs the singularity of the correlation length - must satisfy the inequality $\nu_{\mathrm{d}} \geq 2 / d$, where $d$ is the number of spatial dimensions. Using the hyperscaling relation $\alpha=2-\nu d$, this inequality translates into $\alpha \leq 0$, which in turn implies that there is no divergence in the specific heat. More generally, Harris criterion leads to the conclusion that adding disorder to a pure system with $\nu_{\mathrm{p}} \leq 2 / d$ has a dramatic effect, since it must change the critical exponents, and therefore the universality class of the phase transition.

The influence of disorder on first-order phase transitions has also been considered for a long time. It was soon proposed that impurities tend to reduce the discontinuities of the first derivatives of the free energy (jump of the magnetization, latent heat). It was argued that these discontinuities could even disappear in some cases $\stackrel{4}{\underline{4}}$ Again disorder changes qualitatively the properties of the system, by inducing a second-order phase transition. Later on, this "rounding effect" of disorder was proven to take place in a large class of systems by Aizenman and Wehr ${ }^{5}$ (referred to as AW in the following). Up to now there is no experimental realization of this phenomenon.

It was recently proposed by one of us $\frac{6}{\underline{m}}$ that such a second-order phase transition induced by impurities could actually be studied by experimental means in frustrated magnets such as stacked triangular antiferromagnets $\left(\mathrm{CsMnBr}_{3}, \mathrm{CsMnI}_{3}\right.$, etc. $)$ and rare-earth helimagnets (Ho, Dy, Tb). In the pure case, these materials have been very much studied both experimentally and theoretically since the 1970 s (see Refs. 7 and 8 for re- views). The early calculations indicate a second-order phase transition (see also Ref. 9), in accordance with the observed power-law behavior of thermodynamic quantities. However, there is a wide dispersion of the measured critical exponents. This lack of universality has been recently interpreted as a signature of a weakly first-order phase transition. ${ }^{10.11}$ This issue is still very debated. In any case, we believe that it would be very instructive to study the influence of disorder in such systems. On the one hand, if the transition is of second order in the pure case, one expects a dramatic change of the critical exponents when impurities are added. Following Harris criterion, the exponent $\nu$, which is always found to be smaller than 0.57 , should jump above $2 / 3$. This variation is at least three times bigger than in the case of Ising magnets and might be easier to observe experimentally. On the other hand, if the transition is weakly of first order, it is expected to be turned into a continuous one in the presence of impurities, on the basis of AW. As a consequence, one would observe universal critical exponents, in contrast with the scattered values reported in the pure case. This would provide the first experimental realization of the rounding effect of disorder. This last scenario is supported by a recent nonperturbative renormalization-group calculation in $d=3 \underline{\underline{6}}^{6}$ Actually, the results of AW are not restricted to $d=3$, and apply to any dimension lower than four $\frac{17}{1}$ In the present report, we study the rounding effect of disorder within the standard $\epsilon$ expansion. As we shall see, the perturbative results do not coincide with the conclusions of AW, signaling the onset of physical effects, yet to be determined.

In the following, we describe the one-loop result, from which we argue that higher-order contributions are needed in order to get the qualitative behavior of the system. We show that the $\epsilon$ expansion of the fixed-point coupling constants is singular for some physically relevant values of the number of spin components. Using a simple geometrical picture, we explain the origin of such singularities, and recast the perturbative series into a regular form. Our discussion is quite general, and can be applied to a wide class of theories displaying the same qualitative behavior. We then perform a two-loop calculation and analyze the effect of disorder using this method.

Let us first describe the Hamiltonian which is used in the following. The theoretical studies of the phase transition in the materials considered here started in the 
$1970 \mathrm{~s}, 12,13$ It was then realized that the relevant order parameter is not a simple vector (as for a ferromagneticparamagnetic phase transition), but a set of two vectors $\vec{\varphi}_{1}$ and $\vec{\varphi}_{2}$. This is a direct consequence of frustration, which makes the ground state more degenerate than in the corresponding nonfrustrated system. In physically relevant situations, $\vec{\varphi}_{1}$ and $\vec{\varphi}_{2}$ are taken to have two or three components, depending on the anisotropies of the crystal. Here we consider a direct generalization where the order parameter is composed of $m$ vectors, each having $n$ spin components, and we gather these into a $n \times m$ matrix: $\phi=\left(\vec{\varphi}_{1}, \cdots, \vec{\varphi}_{m}\right)$. Considering only the relevant " $\varphi$-like" terms, the hamiltonian reads:

$$
\mathcal{S}(\phi)=\int d^{d} x\left[\frac{Z}{2} \operatorname{Tr}\left(\partial^{t} \phi . \partial \phi\right)+r \frac{\rho}{2}+\frac{u_{1}}{8} \rho^{2}+\frac{u_{2}}{4} \tau\right]
$$

where $\rho=\operatorname{Tr}\left({ }^{t} \phi \phi\right)$ and $\tau=\operatorname{Tr}\left({ }^{t} \phi \phi{ }^{t} \phi \phi\right)$. Adding impurities induces a spatial dependence of the coupling constants, through the local density of impurities. Using the replica trick, it is, however, possible to average over disorder, and to study the properties of the system by considering a translational invariant Hamiltonian. The price to pay is that we now have to deal with $o$ replicas of the field, and to take the limit $o \rightarrow 0$ at the end of the calculation. The $\varphi^{4}$-like hamiltonian then reads ${ }^{6}$

$$
\widetilde{\mathcal{S}}\left(\left\{\phi_{k}\right\}\right)=\sum_{l=1}^{o} \mathcal{S}\left(\phi_{l}\right)+\frac{u_{3}}{8} \int d^{d} x\left[\sum_{l=1}^{o} \rho_{l}\right]^{2},
$$

where $l$ indices the different copies of the field. Note that only the region $u_{1} \geq 0, m u_{1}+2 u_{2} \geq 0, u_{3} \leq 0$ is of physical relevance ${ }^{1.13}$

Let us describe the situation at the one-loop level. The $\beta$ functions were derived in Ref. [6, and can be read off on Eq. (5) below. Out of the eight solutions to the set of three equations $\beta_{i}=0$, only four are of interest here. The situation of pure frustrated magnets is recovered when $u_{3}=0$. In this plane, we retrieve the two associated fixed points, noted $P^{+}$and $P^{-}$in the following. In addition to these well-known fixed points, we find two new ones which are of particular interest in our study, and which we call $D^{+}$and $D^{-}$. Let us discuss the behavior of the fixed point when $n$ is varied, begining with $P^{+}$and $P^{-}, \frac{12}{2}$ For large values of $n$, they have real coordinates in coupling-constant space. When $n$ is decreased, they approach each other, and coincide at the critical value $n_{\mathrm{p}, 0}^{+}=4(3+\sqrt{6}) \simeq 21.8$ (throughout this report, the numerical values are given for the physically interesting case $m=2$ ). Below $n_{\mathrm{p}, 0}^{+}$, the two fixed points get complex coordinates and are not physical anymore. Finally, when $n$ reaches $n_{\mathrm{p}, 0}^{-}=4(3-\sqrt{6}) \simeq 2.2, P^{+}$and $P^{-}$ reappear, however, not in a region of physical interest. These features follow directly from the expression of the coupling constants at $P^{ \pm}$, which read $(i=1,2,3)$

$$
u_{i}^{ \pm}=\epsilon A_{i}(n) \pm \epsilon B_{i}(n) \sqrt{n-n_{\mathrm{p}, 0}^{-}} \sqrt{n-n_{\mathrm{p}, 0}^{+}} .
$$

We observe the very same qualitative behavior for the couple $D^{ \pm}$, and we denote by $n_{\mathrm{d}, 0}^{ \pm}$the associated critical values of $n$. Surprisingly, the two couples of fixed points $P^{ \pm}$and $D^{ \pm}$annihilate for the same values of $n$ (namely, $n_{\mathrm{p}, 0}^{ \pm}=n_{\mathrm{d}, 0}^{ \pm} \equiv n_{0}^{ \pm}$), and at the same location in couplingconstant space. In the following, we focus our attention on the vicinity of the upper critical value $n_{0}^{+}$, the only physically interesting one, and we drop the superscript.

In summary, at one loop, when $n>n_{0}$, the fixed point $P^{+}$is stable, and the disorder is irrelevant. For $n<n_{0}$, there is no stable fixed point, and the phase transition is likely to be of first order. This is very astonishing, being difficult to reconcile with the general results of AW. Thus one can expect that higher-order corrections will resolve this mismatch. In fact, we expect a qualitative change already at two loops. Indeed, for the pure frustrated magnets, at the value of $n$ where the two fixed points $P^{ \pm}$ meet, one has: ${ }^{14} \nu=2 / d-\epsilon^{2}(\sqrt{6}-1) / 200+o\left(\epsilon^{3}\right)<2 / d$. Using Harris criterion, we conclude that the disorder should be relevant, at least for this value of $n$. This in turn suggests that the degeneracy observed at one loop should be resolved at higher orders, namely, that the two couples of fixed points $P^{ \pm}$and $D^{ \pm}$should annihilate independently and for different values of $n$.

Before embarking in higher-loop calculations, it is useful to do a short digression and to consider the phenomenon of annihilation of fixed points on more general grounds. For the sake of simplicity, consider a theory with two coupling constants $u_{1}$ and $u_{2}$. The points where the associated $\beta$ functions vanish form two curves in the plane $\left(u_{1}, u_{2}\right)$, whose intersections are the fixed points. When a parameter is varied - in our case the number of spin components - these curves move, and so do their intersections. A typical situation is represented in Fig. [1 where one sees the annihilation of two fixed points for $n=n_{c}$. This qualitative picture is already observed at

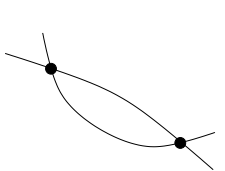

(a) $n>n_{c}$

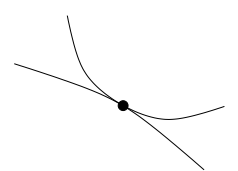

(b) $n=n_{c}$

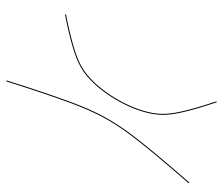

(c) $n<n_{c}$
FIG. 1: Annihilation of two fixed points at $n=n_{c}$. The lines represent zeros of the $\beta$ functions and their intersections represent the fixed points.

one loop and it is clear that it does not change when higher-order corrections are included: the curves of Fig. 1are only slightly displaced. As a consequence, the position $\left(u_{1}^{\star}, u_{2}^{\star}\right)$ where the two fixed-points meet, as well as the corresponding value $n_{c}$ of the number of spin components, receive small corrections at each order of perturbation theory and therefore depend on $\epsilon$. From these considerations, it is an easy matter to deduce the generic form of the fixed-point coordinates near $n_{c}(\epsilon)$. Expanding the $\beta$ functions in coupling constants up to second order around $u_{i}^{\star}$, one is left with quadratic expressions as in the one-loop case. The coordinates of the fixed 
points then read

$$
u_{i}^{ \pm}=\epsilon R_{i}(n, \epsilon) \pm \epsilon S_{i}(n, \epsilon) \sqrt{n-n_{c}(\epsilon)},
$$

where $R_{i}$ and $S_{i}$ are polynomials in $\epsilon$. The previous formula is very appealing when compared with the oneloop form (3) in the vicinity of $n_{0}^{+}$, the main difference $\frac{18}{18}$ being the $\epsilon$ dependence of the critical value of $n$. The whole previous argument is very general, and only makes use of the geometrical picture of Fig. 1] In particular, it does not rely on perturbation theory and Eq. (4) is, in this sense, a nonperturbative result. In the following, we make extensive use of Eq. (4) which, when combined with perturbation theory, provides a very powerful tool.

Let us finally make contact with the usual $\epsilon$ expansion of the fixed-point coupling constants, the coefficients of which are found to be singular in the limit $n \rightarrow n_{0} \underline{14}$ We can easily understand the origin of such singularities in the light of our previous discussion: writing $n_{c}(\epsilon)=$ $n_{c, 0}+\epsilon n_{c, 1}+\cdots$ and expanding Eq. (44) in powers of $\epsilon$, one gets terms of the form $\epsilon^{\ell} /\left(n-n_{0}\right)^{m-1 / 2}$, with $m \leq \ell-1$. Therefore, the regular form (4) corresponds to a resummation of infinitely many singular terms. In practice, one reconstructs the polynomials $R_{i}$ and $S_{i}$ by matching the $\epsilon$ expansion of Eq. (4) for $n \neq n_{0}$ with the corresponding perturbative series.

Comming back to the problem at hand, we now discuss the expected influence of higher-loop corrections on the one-loop flow diagram. The critical values of $n$ introduced before, now depend on $\epsilon$, and we shall refer to them as $n_{\mathrm{p}}(\epsilon)$ and $n_{\mathrm{d}}(\epsilon)$. As already explained, Harris
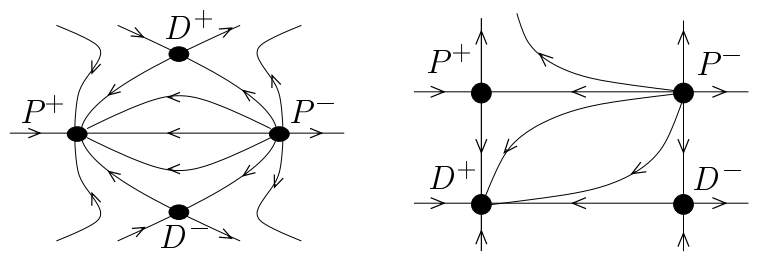

FIG. 2: Flow diagram above and below $n_{\mathrm{s}}$. The fixed point $D^{+}$crosses the plane $u_{3}=0$ through $P^{+}$, and gets stable afterward.

criterion indicates that disorder should be relevant, at least for $n=n_{\mathrm{p}}(\epsilon)$. This can only happen if, for some particular value $n_{\mathrm{s}}(\epsilon)$, either $D^{+}$or $D^{-}$crosses the plane $u_{3}=0$ through $P^{+}$, and exchanges its stability with it. Moreover, following AW, we expect that for $n<n_{p}(\epsilon)$ the phase transition is governed by a disordered fixed point. This favors the first scenario, which is depicted in Fig. 2. In addition to $n_{\mathrm{p}}(\epsilon), n_{\mathrm{d}}(\epsilon)$, and $n_{\mathrm{s}}(\epsilon)$, we shall be interested in a fourth particular value of $n$, noted $n_{\mathrm{H}}(\epsilon)$, for which the critical exponent $\nu$ associated with $P^{+}$just equals $2 / d$. Exploiting the Harris criterion and the topology of the flow, we see that $n_{\mathrm{s}}$ is larger than $n_{\mathrm{p}}, n_{\mathrm{d}}$, and $n_{\mathrm{H}}$.

With this in mind, we perform a two-loop calculation of the $\beta$ functions. Using dimensional regularization and minimal subtraction, we get

$$
\begin{aligned}
& \beta_{1}=-u_{1} \epsilon+2 v_{4}\left\{u_{1}^{2} I_{8,0,1}+4 u_{1}\left(3 u_{3}+u_{2} I_{1,1,0}\right)\right. \\
& \left.+12 u_{2}^{2}\right\}-4 v_{4}^{2}\left\{3 u_{1}^{3} I_{14,0,3}+2 u_{1}^{2}\left(22 u_{2} I_{1,1,0}\right.\right. \\
& \left.+u_{3} I_{58,0,11}\right)+u_{1}\left(68 u_{2} u_{3} I_{1,1,0}+2 u_{2}^{2} I_{87,5,5}\right. \\
& \left.\left.+u_{3}^{2} J_{82,5}\right)+24 u_{2}^{2}\left(6 u_{3}+u_{2} I_{3,1,0}\right)\right\}, \\
& \beta_{2}=-u_{2} \epsilon+4 u_{2} v_{4}\left\{\left(6 u_{1}+u_{2} I_{4,1,0}+6 u_{3}\right\}\right. \\
& -4 u_{2} v_{4}^{2}\left\{6 u_{2}^{2} I_{17,3,1}+4 u_{2}\left(u_{1}+u_{3}\right) I_{29,11,0}\right. \\
& \left.+u_{1}\left(u_{1}+2 u_{3}\right) I_{82,0,5}+u_{3}^{2} J_{82,5}\right\}, \\
& \beta_{3}=-u_{3} \epsilon+2 u_{3} v_{4}\left\{2 u_{1} I_{2,0,1}+4 u_{2} I_{1,1,0}\right. \\
& \left.+u_{3} J_{8,1}\right\}-4 u_{3} v_{4}{ }^{2}\left\{10 u_{2}^{2} I_{3,1,1}+3 u_{3}^{2} J_{14,3}\right. \\
& \left.+4 u_{2}\left(5 u_{1}+11 u_{3}\right) I_{1,1,0}+u_{1}\left(5 u_{1}+22 u_{3}\right) I_{2,0,1}\right\},
\end{aligned}
$$

where $v_{4}=1 /\left(32 \pi^{2}\right), I_{i, j, k}=i+j(m+n)+k(m n)$, and $J_{i, j}=i+j(m n o)$. We also give the expressions of the critical exponents:

$$
\begin{aligned}
& \eta=2 v_{4}^{2}\left\{I_{2,0,1} u_{1}\left(u_{1}+2 u_{3}\right)+4 I_{1,1,0} u_{2}\left(u_{1}+u_{3}\right)\right. \\
& \left.\quad+2 I_{3,1,1} u_{2}^{2}+2 J_{2,1} u_{3}^{2}\right\} \\
& 1 / \nu=2-2 v_{4}\left\{I_{2,0,1} u_{1}+2 I_{1,1,0} u_{2}+J_{2,1} u_{3}\right\}+5 \eta
\end{aligned}
$$

By using Eq. (4), we can safely study our expressions near $n=n_{0}$. The first surprising result is that the four values of $n$ introduced above are still degenerate at order $\epsilon$, i.e. $n_{c, 1} \equiv n_{1}=-2(18+7 \sqrt{6}) / 3 \simeq-23.4$, for $c=$ $\mathrm{d}, \mathrm{p}, \mathrm{s}, \mathrm{H}$. In principle, the next correction, i.e. $n_{c, 2}$, is obtained by a three-loop calculation. However, due to Eq. (44) we can show that the degeneracy is resolved at order $\epsilon^{2}$ without performing explicitly a three-loop calculation. Let us illustrate this point on a simple case. When $n=n_{\mathrm{s}}(\epsilon)$, the fixed points $P^{+}$and $D^{+}$coincide and the coupling constant $u_{3}$ of $D^{+}$vanishes. Inserting $n=n_{\mathrm{s}}(\epsilon)=n_{0}+\epsilon n_{1}+\epsilon^{2} n_{\mathrm{s}, 2}$ in Eq. (4), and expanding the equation $u_{3}^{+}=0$ to second order in $\epsilon$, one obtains

$$
\sqrt{n_{\mathrm{s}, 2}-n_{\mathrm{d}, 2}}=-\frac{\left.\left(\partial_{\epsilon}+n_{1} \partial_{n}\right) R_{3}(n, \epsilon)\right|_{n=n_{0}, \epsilon=0}}{S_{3}\left(n_{0}, 0\right)} .
$$

The expressions appearing in the above equation involve only two-loop expressions and can therefore be computed here. For $m=2$, we find

$$
n_{\mathrm{s}, 2}-n_{\mathrm{d}, 2}=(1092+463 \sqrt{6}) / 50 \simeq 44.5 .
$$

Using the same technique for the equations $u_{i}\left(D^{+}\right)=$ $u_{i}\left(P^{+}\right)$at $n=n_{\mathrm{s}}(\epsilon)(i=1,2)$, as well as $\nu\left(P^{+}\right)=2 / d$ at $n=n_{\mathrm{H}}(\epsilon)$, we get

$$
n_{\mathrm{s}, 2}-n_{\mathrm{p}, 2}=n_{\mathrm{H}, 2}-n_{\mathrm{p}, 2}=(7 \sqrt{6}-12) / 50 \simeq 0.103 .
$$

We see that $n_{\mathrm{s}}(\epsilon)=n_{\mathrm{H}}(\epsilon)$ up to second order: the Harris criterion is satisfied precisely at this value of $n$ where disorder becomes relevant. We mention that a three-loop calculation for the pure $\left(u_{3}=0\right)$ frustrated magnets 14 yielded the value: $n_{\mathrm{p}, 2} \simeq 7.09$. Combining this with our 
"two-loop" results (8) and (9), we are able to compute the "three-loop" contributions: $n_{\mathrm{d}, 2}=-37.3, n_{\mathrm{s}, 2}=$ $n_{\mathrm{H}, 2}=7.19$. We find the same features for any value of $m$, except for the numerical results. For example, for $m=3$, we get $n_{\mathrm{d}, 2}=-48.2$ and $n_{\mathrm{s}, 2}=n_{\mathrm{H}, 2}=11.1$.

We summarize our results in Fig. 3. There are essen-

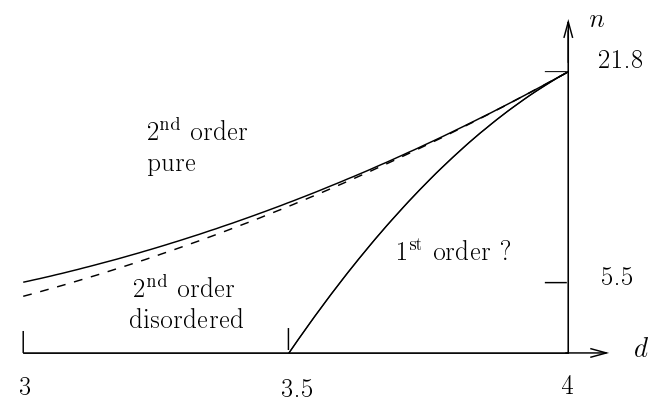

FIG. 3: Different types of phase transition in the $(n, d)$ plane. The lines represent $n_{\mathrm{S}}(\epsilon), n_{\mathrm{p}}(\epsilon)$ and $n_{\mathrm{d}}(\epsilon)$ from top to bottom. The splitting between $n_{\mathrm{S}}(\epsilon)$, and $n_{\mathrm{p}}(\epsilon)$ has been magnified

tially three distinct regions in the $(n, d)$ plane. In the first one $\left[n>n_{\mathrm{s}}(\epsilon)\right]$, the transition is of second order and is governed by the pure fixed point $P^{+}$. In the second one $\left[n_{\mathrm{d}}(\epsilon)<n<n_{\mathrm{S}}(\epsilon)\right]$, the disorder is relevant and the system undergoes a second-order phase transition governed by the fixed point $D^{+}$. This region is divided by the curve $n_{\mathrm{p}}(\epsilon)$ (dotted line in Fig. [3). Above it, $P^{+}$still exists, but is unstable against disorder, as expected from the Harris criterion. Below, the pure fixed point has disappeared: the fluctuation-driven first-order phase transition observed in the pure system is turned into a continuous one by impurities, in line with AW. In the whole second region, the disorder induces a new universality class, and therefore new critical exponents. For instance, on the line $n_{\mathrm{d}}(\epsilon)$, one has $\eta=\epsilon^{2} / 48$ and $\nu=2 / d+\epsilon^{2}(13+7 \sqrt{6}) / 200$ up to $o\left(\epsilon^{3}\right)$. We note that the experimentally relevant cases $(d=3, n=2,3)$ belong to this region. Although our results can only serve as a rough indication in $d=3$, they are corroborated by the conclusions of a nonperturbative approach, and support the recent proposal to study experimentally the rounding effect of disorder in frustrated magnets 6 Finally, there is a third region $\left[n<n_{\mathrm{d}}(\epsilon)\right]$, where we find no physically relevant stable fixed point: here, the disorder does not counterbalance the effect of fluctuations, and the phase transition most probably remains of first order. We stress that for $n<n_{0}$, the deep perturbative regime $\epsilon \ll 1$, where our approach is under control, belongs to this third region.

If the results found in the first two regions are well understood in terms of the Harris criterion and AW, the situation is still puzzling in the third region. Indeed, perturbation theory predicts a discontinuous phase transition where AW state that the latent heat vanishes, which in turn strongly indicates a continuous transition. We suggest three possible scenarios which could explain this mismatch. It could happen that, although the latent heat vanishes, the phase transition is still of first $\operatorname{order}^{19}$. However, simple Landau-type arguments indicate that this is not very likely. Another possibility is that the replica symmetry, which was implicitly assumed to hold, may actually be broken. This could be investigated following the lines of Ref. 15. Finally, the expected second-order phase transition could be governed by a nonperturbative fixed point, unreachable by the approach we considered here. These three possibilities are very interesting and deserve more investigations.

We thank B. Delamotte and D. Mouhanna for discussions. M. T. acknowledges financial support from Marie Curie Fellowship Association under contract No. HPMFCT-2001-01343.
* Electronic address: serreau@thphys.uni-heidelberg.de

$\dagger$ Electronic address: tissier@thphys.uni-heidelberg.de

1 R. Folk, Y. Holovatch, and T. Yavors'kii, Uspiekhi Fizichieskikh Nauk 173, 175 (2003) [cond-mat/0106468].

2 A. B. Harris, J. Phys. C 7, 1671 (1974).

3 J. T. Chayes, L. Chayes, D. S. Fisher, and T. Spencer, Phys. Rev. Lett. 57, 2999 (1986).

4 Y. Imry and M. Wortis, Phys. Rev. B 19, 3580 (1979).

5 M. Aizenman and J. Wehr, Phys. Rev. Lett 62, 2503 (1989), erratum ibid 64, 1311 (1990).

6 M. Tissier (2002), cond-mat/0203370.

7 M. F. Collins and O. A. Petrenko, Can. J. Phys. 75, 605 (1997).

8 H. Kawamura, J. Phys. C 10, 4707 (1998).

9 A. Pelissetto, P. Rossi, and E. Vicari, Phys. Rev. B 63, 140414 (2001).

10 M. Itakura, J. Phys. Soc. Jpn. 72, 74 (2003).

11 M. Tissier, B. Delamotte, and D. Mouhanna, to appear in
Phys. Rev. B [cond-mat/0107183].

12 T. Garel and P. Pfeuty, J. Phys. C: Solid St. Phys. 9, L245 (1976).

13 M. Yosefin and E. Domany, Phys. Rev. B 32, 1778 (1985).

14 A. Pelissetto, P. Rossi, and E. Vicari, Nucl. Phys. B [FS] 607, 605 (2001).

15 E. Brézin and C. D. Dominicis, Eur. Phys. J. B 19, 467 (2001).

16 D. J. Gross and I. K. H. Sompolinsky, Phys. Rev. Lett. 55, 304 (1985).

17 Strictly speaking, this holds if the number of spin components is larger than two

18 We mention that in principle one can repeat this construction near $n=n_{0}^{-}$, so as to get a form with two square roots, similar to (3).

19 There could be a jump in the magnetization, as observed in Ref. 16. 